\title{
Functional properties of metallomesogens modulated by molecular and supramolecular exotic arrangements
}

\author{
Alessandra Crispini ${ }^{1}$, Mauro Ghedini ${ }^{2}$ and Daniela Pucci ${ }^{* 2}$
}

\section{Address:}

${ }^{1}$ Centro di Eccellenza CEMIF.CAL-LASCAMM, CR-INSTM Unità della Calabria, Dipartimento di Scienze Farmaceutiche, Università della Calabria, Edificio Polifunzionale, Arcavacata di Rende (CS), 87036, Italy and ${ }^{2}$ Centro di Eccellenza CEMIF.CAL-LASCAMM, CR-INSTM Unità della Calabria, Dipartimento di Chimica, Università della Calabria, Via P. Bucci Cubo 14C, Arcavacata di Rende (CS), 87036, Italy

Email:

Daniela Pucci - d.pucci@unical.it

* Corresponding author

Keywords:

coordination complexes; functionality; liquid crystals;

metallomesogens
Beilstein Journal of Organic Chemistry 2009, 5, No. 54 doi: $10.3762 /$ bjoc. 5.54

Received: 01 July 2009

Accepted: 02 October 2009

Published: 12 October 2009

Guest Editor: S. Laschat

(c) 2009 Crispini et al; licensee Beilstein-Institut. License and terms: see end of document.

\begin{abstract}
New concepts for the synthesis of metallomesogens have been recently developed in order to use the metal centre as a scaffold for grafting different functionalities and inducing non-conventional shapes and properties in the resulting complexes. Our strategy was based on the synthesis of mesogenic coordination complexes whose molecular architectures are controlled by the modulation of different and tunable molecular motifs: the nature of the metal ion and the surrounding ligands as central unit, the number of flexible chains at the periphery, and the nature of counter-ions in ionic complexes. The appropriate choice of molecular construction motifs allows control at global architectures and induces pre-selected properties from the level of single molecule to supramolecular network, confirming that metal coordination provides a helpful tool for obtaining multifunctional soft materials.
\end{abstract}

\section{Introduction}

Recent interest in designing novel soft and functional materials with more and more challenging requirements such as improved charge transport, luminescence, chirality and biological functions for high-tech applications has been directed towards the use of new mesomorphic systems [1-14]. Design principles based only on the shape and the symmetry of the mesogenic molecules is giving way to alternative concepts for achieving new molecular and supramolecular motifs able to give rise to dynamic functional properties and unusual topologies and families of mesophases. This goal has been reached through different strategies: the creation of hybrid molecular topologies [15-22]; the micro-segregation between incompatible units within molecules [15,23-25]; the development of self-organizing super and supramolecules able to generate complex hier- 
archical structures through specific inter- or intramolecular interactions [25-30]. The high level of functionality integrated into molecular-based electronic systems obtained by incorporating metal centres into selected organic structures [31] supports the design of metal-containing liquid crystals (metallomesogens) as an effective and helpful way to expand of the traditional range of technological applications of liquid crystals [32-37].

We have been involved for long time in the field of metallomesogens with the synthesis of cyclopalladated rod-like complexes starting from mesogenic azo and azoxybenzenes, confirming that mesomorphism of the organic precursors is preserved after complexation [38]. More recently, our work has addressed the design of new, higher performing complexes, whose structures, inaccessible for organic liquid crystalline systems, are able both to induce very low transition temperatures and to modulate their optical, electronic and thermal properties [34,36,38]. A multi-motif approach based on the spontaneous association of single tectons such as the metal-ligand central unit, the number of flexible chains at the periphery, the type of complementary ligands occupying the coordination sphere of the metal ion and the counter-ion in ionic systems, has been followed. In this paper selected examples of recently synthesised multifunctional metallomesogens are highlighted, all obtained from classical and unusual nitrogen ligands and different metal centres from across the periodic table, going from the most common $\mathrm{Pd}(\mathrm{II})$ to the scarcely used $\mathrm{Pt}(\mathrm{II})$ and Zinc(II), until the never used Ga(III). Indeed, with the nature of the metal centre being the leading actor in the design of new metallomesogens, the choice of unexplored metal centres is an effective route to a new generation of dynamically multifunctional soft materials, with higher performance than classical liquid crystals. Our interest in the synthesis of metallomesogens and their wide use in the field of material science, coupled with the fact that transition metal complexes have potential antitumor activity, led us to believe that a great number of metal-containing liquid crystals, already synthesized and analysed with respect to their chemical and physical properties, can constitute a huge database for the design of new biologically relevant complexes.

\section{Review}

\section{Palladium(II) complexes}

New mononuclear ortho-palladated complexes have been prepared in order to expand the applications of these systems towards new peculiar properties induced by innovative cyclometallating and ancillary ligands.

For example, an interesting red emitting mesomorphic complex has been prepared starting from the Nile red dye
(9-diethylamino-5H-benzo $[a]$ phenoxazine-5-one) as a cyclometallating ligand and from the suitably functionalized curcumin $\beta$-diketonate as a complementary $\mathrm{O}, \mathrm{O}$ chelating ligand (Figure 1).

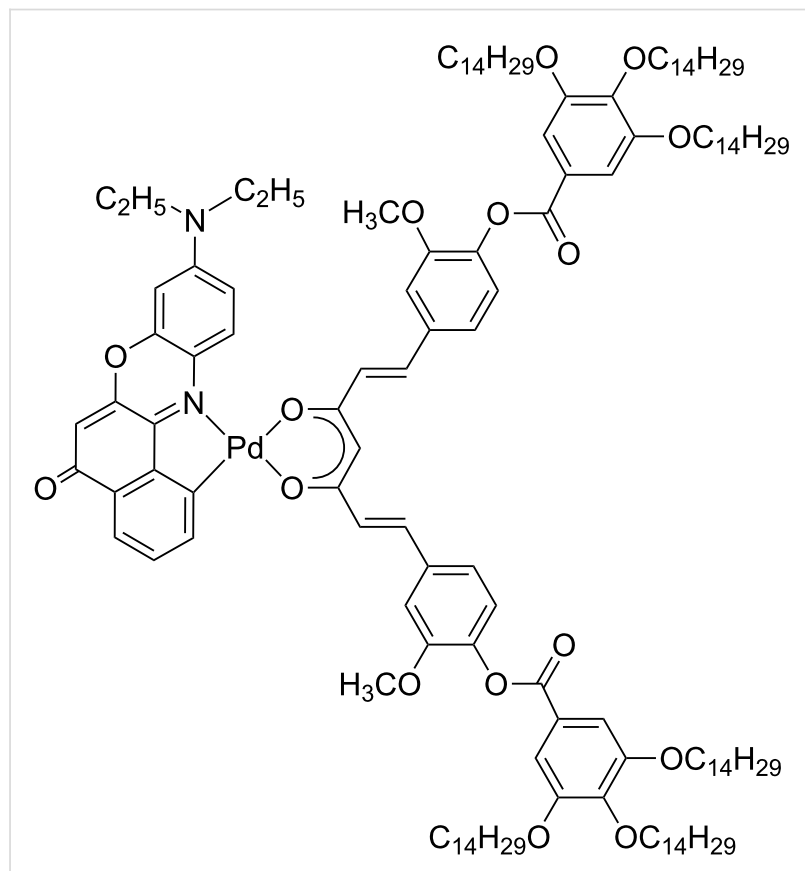

Figure 1: Molecular structure of NIRPAC: a Pd(II) complex based on Nile red and a curcumin derivative.

The presence of the principal ligand [39-44] introduces interesting luminescence properties into the metallic system (which is luminescent in solution), in the red region of the Vis spectrum $(\lambda=610 \mathrm{~nm})$, with emission quantum yields in the range of $6-23 \%$, depending on the solvent. However, the $3,4,5-$ trialkoxy-substituted benzoato fragment of the curcumin ligand imposes an overall hemi-disc structure to the resulting palladium derivative [45] allowing the onset, even at room temperature, of a rectangular columnar mesophase which is stable over a very large temperature range $\left(140^{\circ} \mathrm{C}\right)$. The emission properties observed in the solution are preserved in the liquid crystalline state, therefore the flat disk-like molecular structure organized into columns, the broad thermal stability and the luminescence in the red region of the visible spectrum make this new Pd(II) complex a very intriguing candidate for applications in OLED devices.

A further class of ortho-palladated complexes has been obtained starting from the 2-phenylquinoline, a ligand extensively used in the synthesis of cyclometallated iridium(III) and platinum(II) derivatives $[46,47]$ but whose reactivity towards $\mathrm{Pd}(\mathrm{II})$ centres is unexplored. This kind of ligand has been functionalised with a chiral group such as a cholesteryl ester unit, introduced as 
terminal substituent in 4 position, and chosen for many different purposes, including its universal affinity for cell membranes and its ability to self order into liquid crystalline state [48]. The functionalised 2-phenylquinoline ligand has firstly been cyclopalladated and then conjugated to a number of $\mathrm{O}, \mathrm{O}$ chelating $\beta$-diketonate ligands, giving rise to a series of mononuclear complexes combining two chelating functional moieties in their structures (Figure 2) [49].

The coordination to the Pd(II) centre induces, in all the resulting compounds, thermotropic mesomorphism whose nature has been found to be strictly related to the ancillary ligand. Indeed a transition from a calamitic to a columnar mesophase is observed, through a calamitic/discotic cross-over point, due to the peculiar combination of two different molecular architectures. The mononuclear tropolonate derivative (A in Figure 2) shows a chiral nematic phase while the half-disc-shaped curcuminoids (B in Figure 2) self-assemble with the formation of columnar mesophases.

Moreover, the presence of biologicaly active fragments (the $\mathrm{O}, \mathrm{O}$ chelating ligands) induces promising anticancer activity in vitro against two human prostatic cancer cell lines in all these complexes suggesting that, through the careful choice of the molecular building blocks, cyclopalladated mesogens represent multifunctional biomaterials. They bear at the same time the active principle and the membrane-compatible delivery component, and are becoming innovative tools in establishing new and effective anticancer therapies.

Finally, in order to investigate the unexplored cyclopalladating ability of 2,2'-pyridylpyrroles, a series of 3,5-disubstituted-2,2'pyridylpyrroles and their mononuclear Pd(II) ortho-palladated

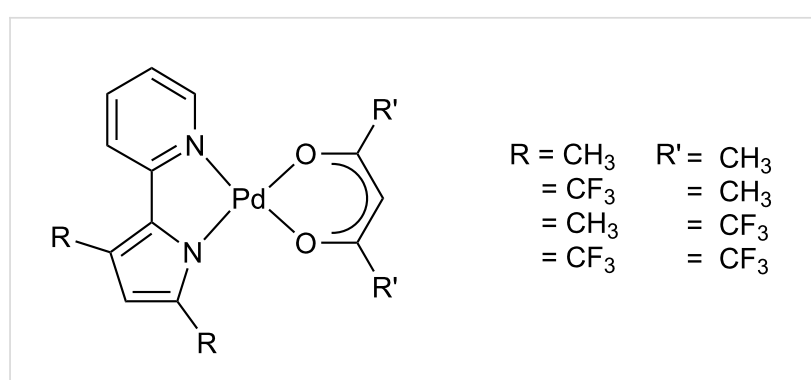

Figure 3: Some unusual palladiomesogens based on 3,5disubstituted-2,2'-pyridylpyrroles and $\beta$-diketonates.

derivatives (Figure 3) have been prepared [50].

The resulting complexes, containing as ancillary ligands some acetylacetonates, are "non conventional" shaped palladiomesogens. Indeed, they completely lack terminal tails, usually necessary for inducing mesomorphism. In this case the presence of fluorinated groups on the complementary ligands promotes a delicate balance between incompatible parts of the molecules, generating phase segregated structures favourable for the appearance of mesomorphism through hexagonal columnar phases. The strategy adopted, building on the modulation of incompatible simple synthons on a metallomesogenic molecule, opens new possibilities for tailoring soft materials with nonconventional structures.

\section{Platinum(II) and Zinc(II)}

For the less explored $\mathrm{Pt}(\mathrm{II})$ and $\mathrm{Zn}(\mathrm{II})$ metal centres versatile 2,2'-bipyridines have been selected since they are well-known building blocks for the formation of inorganic functional nanomaterials [51]. The complexation of non-mesogenic 4,4'-disubted 2,2'-bipyridine ligands with Pt(II) salts confirmed the role of

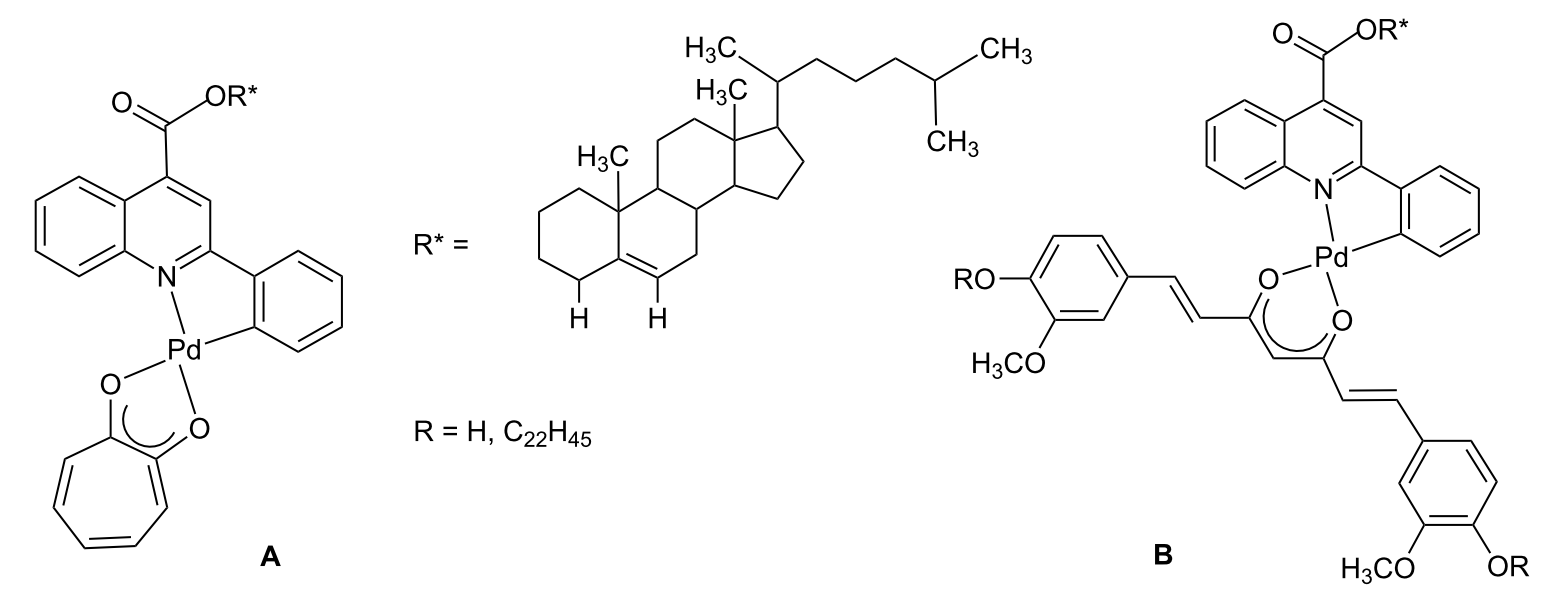

Figure 2: Molecular structure of $\mathrm{Pd}(\mathrm{II})$ complexes based on functionalised 2-phenylquinolines and $\beta$-diketonates. 
coordination chemistry in the metal-mediated formation of liquid crystals. Indeed the induction of a dipole moment upon coordination with an $\mathrm{MX}_{2}$ moiety, allowed most of the half-disc shaped complexes [ $\mathrm{L}^{\mathrm{n}} \mathrm{PtX} \mathrm{X}_{2}$ (Figure 4) to self-assemble into full disc shaped dimers as described by the complementary shape approach [52].

The appearance of mesomorphism is related to the length of the alkyl chains: indeed the complexes based on the 2,2'-bipyridines with short tails melted directly into an isotropic liquid, while the higher homologues produce the global rod-like shape responsible for the liquid crystalline behaviour, namely of a lamello-columnar type. Changes of the ancillary ligands have been carried out in order to use dipole coupling as a tool for molecular architecture. Mesomorphic behaviour was found to depend on the size of the $\mathrm{X}$ group and on the dipole moment associated with the Pt-X bond, with the sequence, for the clearing points $\mathrm{Cl}<\mathrm{Br}<\mathrm{I}$ and the azide group which promoted a lowering of both transition temperatures. Moreover, these $\mathrm{Pt}(\mathrm{II})$ complexes revealed to be photoluminescent with a good degree of tunability, depending on the $\pi$-donor capacity of the $\mathrm{X}$ ligands.

Zinc(II) complexes are widely applied in OLED technology for their light emitting efficiency, high thermal and redox stability, and tunable electronic properties [53-55]. Hence the design of $\mathrm{Zn}$ (II) complexes showing at the same time, order, mobility, and changes in molecular organization in response to external stimuli could be a good strategy for developing new soft materials for innovative applications. In this context the same 2,2'-bipyridines have been coordinated to $\mathrm{Zn}$ (II) ions where the tetrahedral geometry of the $\mathrm{Zn}$ (II) derivatives prevented the single molecules self-assembling in dimers. Moreover, strong intermolecular contacts stabilized the crystalline state and no mesomorphic behaviour was observed below the melting point [52]. We decided to extend this work changing the substituents on the

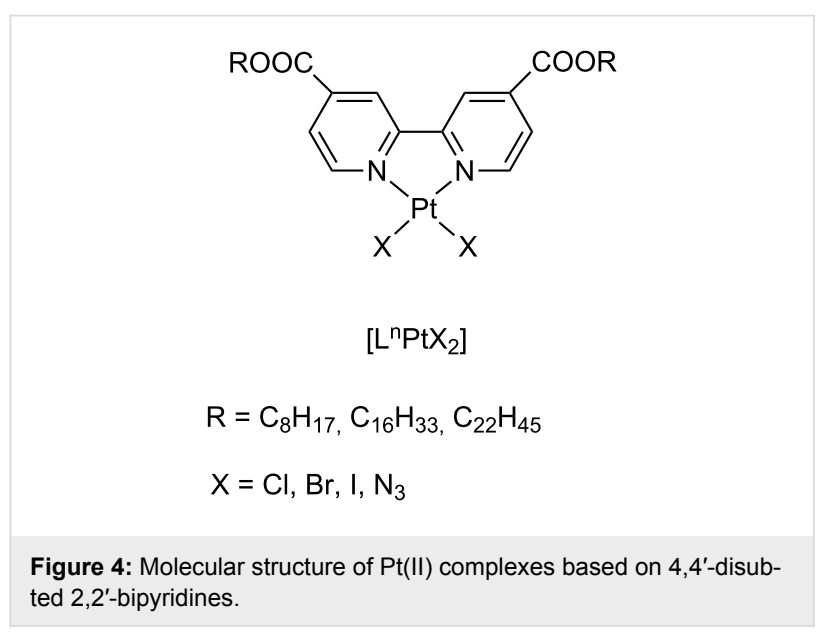

bipyridine ligands by introducing of further aromatic rings equipped with several aliphatic tails each. Hence the synthesis of a series of cis-dichloro hexacatenar $\mathrm{Zn}$ (II) complexes has been performed (Figure 5) [56].

In this case the molecular organization in the mesophase, mainly driven by intermolecular attractive interactions (hydrogen bonds, $\mathrm{C}-\mathrm{H}-\pi$ and $\pi-\pi$ contacts) between the large flat aromatic cores rather than dipolar or metal-metal interactions, is able to produce supramolecular columnar mesomorphism, appearing for the first time in tetrahedral $\mathrm{Zn}(\mathrm{II})$ derivatives.

These results show more and more that, by careful choice of molecular building blocks, it is possible to modulate the interactions necessary for organization of single molecules in to supramolecular architectures to give rise to the desired metallomesogenic material. Moreover, preliminary measurements of photoconductivity on these complexes doped with $\mathrm{C}_{60}$ to increase absorption in the visible region, have given excellent results and further experiments are still in progress.

\section{Gallium (III)}

Since the nature of the metal centre represents an important tool for tailoring specific molecular shapes and topologies, we have selected the $\mathrm{Ga}$ (III) ion, never used before in the design of metallomesogens, in order to explore the possibility of inducing mesomorphism in luminescent metal complexes [57].

In particular, we have explored the possibility of promoting mesomorphism in pentacoordinate bisquinolinate $\mathrm{Ga}$ (III) coordination compounds, well-known blue emitting species, whose properties derive from solid state interactions [58-60]. Hence we have introduced the mobility of a promesogenic polycatenar group through the 3,4,5-tris(tetradecyloxy)-benzoyloxy monodentate ligand in the carboxylate unit, keeping the two<smiles></smiles>

Figure 5: Molecular structure of $\mathrm{Zn}$ (II) complexes based on polycatenar 4,4'-disubstituted 2,2'-bipyridines. 


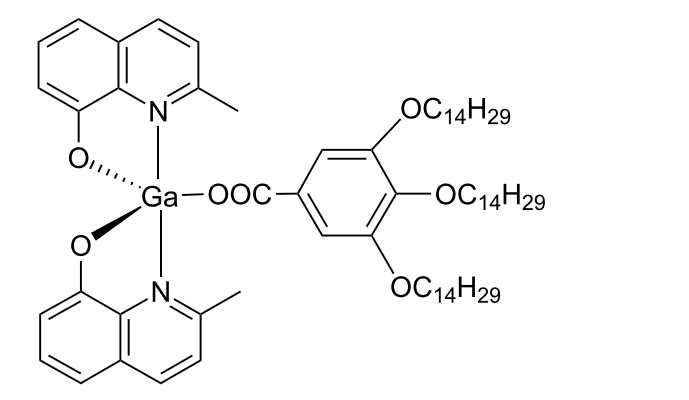

Figure 6: Molecular structure of a gallium(III) mesogen.

quinolinate groups responsible for intermolecular interactions in the crystalline organization grafted around the gallium(III) centre [61]. In this way a unconventional jellyfish shape molecule has been obtained and its molecular structure has been confirmed through single crystal X-ray diffraction (Figure 6).

Despite its unusual molecular shape, this complex shows thermotropic mesomorphism, with a lamello-columnar organization and, at the same time, a light-green emission with the typical high quantum yield of pentacordinated $\mathrm{Ga}$ (III) complexes ( $40 \%$ in solution) [62], has been detected.

\section{Conclusions}

We have selected some examples of recent works on metallomesogens demonstrating that, moving across the periodic table, it is possible to expand the pool of metal ions able to induce mesomorphism in an appropriate framework. Hence it is possible to modulate single molecular geometries starting from the conventional square planar ion $[\mathrm{Pd}(\mathrm{II})]$, and going to the tetrahedral $[\mathrm{Zn}(\mathrm{II})]$ and pentacoordinate $\mathrm{Ga}(\mathrm{III})$ to obtain novel, non conventional structures. Moreover, by changing the nature of coordinating ligands and the kinds of substituents, further properties such as luminescence and bioactivity can be promoted at the same time, leading to smart multifunctional material. Finally, through the appropriate choice of crucial single synthons, it is possible to modulate the role of intermolecular interactions in the resulting architecture in order to create new supramolecular arrays with peculiar properties arising either from individual building blocks or from their synergy.

\section{Acknowledgments}

Financial support received from the Ministero dell'Istruzione, dell'Università e della Ricerca Scientifica (MIUR) through the Centro di Eccellenza CEMIF.CAL (CLAB01TYEF) and the PRIN projects 2006038447 is gratefully acknowledged.

\section{References}

1. Bushby, R. J.; Lozman, O. R. Curr. Opin. Solid State Mater. Sci. 2002, 6, 569-578. doi:10.1016/S1359-0286(03)00007-X

2. Bushby, R. J.; Lozman, O. R. Curr. Opin. Colloid Interface Sci. 2002, 7, 343-354. doi:10.1016/S1359-0294(02)00085-7

3. O'Neill, M.; Kelly, S. M. Adv. Mater. 2003, 15, 1135-1146. doi:10.1002/adma.200300009

4. Hanna, J. Opto-Electron. Rev. 2005, 13, 259-267.

5. lino, H.; Hanna, J. Opto-Electron. Rev. 2005, 13, 295-302.

6. Woliński, T. R.; Ertman, S.; Lesiak, P.; Domański, A. W.; Czapla, A.; Dąbrowski, R.; Nowinowski-Kruszelnicki, E.; Wójcik, J. Opto-Electron. Rev. 2006, 14, 329-334. doi:10.2478/s11772-006-0045-6

7. Palffy-Muhoray, P.; Cao, W.; Moreira, M.; Taheri, B.; Munoz, A. Philos. Trans. R. Soc. London, Ser. A 2006, 364, 2747-2761. doi:10.1098/rsta.2006.1851

8. Ford, A. D.; Morris, S. M.; Coles, H. J. Mater. Today 2006, 9, 36-42. doi:10.1016/S1369-7021(06)71574-7

9. Woltman, S. J.; Jay, G. D.; Crawford, G. P. Nat. Mater. 2007, 6, 929-938. doi:10.1038/nmat2010

10. Goodby, J. W.; Saez, I. M.; Cowling, S. J.; Görtz, V.; Draper, M.; Hall, A. W.; Sia, S.; Cosquer, G.; Lee, S.-E.; Raynes, E. P. Angew. Chem., Int. Ed. 2008, 47, 2754-2787. doi:10.1002/anie.200701111

11. Sergeyev, S.; Pisula, W.; Geerts, Y. H. Chem. Soc. Rev. 2007, 36, 1902-1929. doi:10.1039/b417320c

12. Laschat, S.; Baro, A.; Steinke, N.; Giesselmann, F.; Hägele, C.; Scalia, G.; Judele, R.; Kapatsina, E.; Sauer, S.; Schreivogel, A.; Tosoni, M. Angew. Chem., Int. Ed. 2007, 46, 4832-4887. doi:10.1002/anie.200604203

13. Shimizu, Y.; Oikawa, K.; Nakayama, K.-i.; Guillon, D. J. Mater. Chem. 2007, 17, 4223-4229. doi:10.1039/b705534j

14. Vera, F.; Serrano, J. L.; Sierra, T. Chem. Soc. Rev. 2009, 38, 781-796. doi:10.1039/b800408k

15. Tschierske, C. J. Mater. Chem. 2001, 11, 2647-2671. doi:10.1039/b102914m

16. Goodby, J. W.; Bruce, D. W.; Hird, M.; Imrie, C.; Neal, M. J. Mater. Chem. 2001, 11, 2631-2636. doi:10.1039/b108757f

17. Tschierske, C. Nature 2002, 419, 681-683. doi:10.1038/419681a

18. Amaranatha Reddy, R.; Tschierske, C. J. Mater. Chem. 2006, 16, 907-961. doi:10.1039/b504400f

19. Kato, T.; Hirai, Y.; Nakaso, S.; Moriyama, M. Chem. Soc. Rev. 2007, 36, 1857-1867. doi:10.1039/b612546h

20. Lehmann, M. Chem.-Eur. J. 2009, 15, 3638-3651. doi:10.1002/chem.200802625

21. Marcos, M.; Martín-Rapún, R.; Omenat, A.; Serrano, J. L. Chem. Soc. Rev. 2007, 36, 1889-1901. doi:10.1039/b611123h

22. Imrie, C. T.; Henderson, P. A. Chem. Soc. Rev. 2007, 36, 2096-2124. doi:10.1039/b714102e

23. Kato, T. Science 2002, 295, 2414-2418.

24. Kato, T.; Mizoshita, N. Curr. Opin. Solid State Mater. Sci. 2002, 6, 579-587. doi:10.1016/S1359-0286(03)00006-8

25. Saez, I. M.; Goodby, J. W. J. Mater. Chem. 2005, 15, $26-40$. doi:10.1039/b413416h

26. Tschierske, C. Chem. Soc. Rev. 2007, 36, 1930-1970. doi:10.1039/b615517k

27. Hirai, Y.; Monobe, H.; Mizoshita, N.; Moriyama, M.; Hanabusa, K.; Shimizu, Y.; Kato, T. Adv. Funct. Mater. 2008, 18, 1668-1675. doi:10.1002/adfm.200701313 
28. Kato, T.; Mizoshita, N.; Kishimoto, K. Angew. Chem., Int. Ed. 2006, 45, 38-68. doi:10.1002/anie.200501384

29. Kato, T.; Yasuda, T.; Kamikawa, Y.; Yoshio, M. Chem. Commun. 2009, 729-739. doi:10.1039/b816624b

30. Tam-Chang, S.-W.; Huang, L. Chem. Commun. 2008, 1957-1967. doi:10.1039/b714319b

31. Low, P. J. Dalton Trans. 2005, 2821-2824. doi:10.1039/b506017f

32. Serrano, J. L. Metallomesogens; VCH: Weinheim, 1996.

33. Donnio, B.; Bruce, D. W. In Liquid Crystals II Metallomesogens; Mingos, D. M. P., Ed.; Structure and Bonding, Vol. 95; Springer: Berlin, 1999.

34. Donnio, B.; Guillon, D.; Deschenaux, R.; Bruce, D. W. Metallomesogens. In Comprehensive Coordination Chemistry II; McCleverty, J. A.; Meyer, T. J., Eds.; Elsevier: Oxford, 2003; Vol. 6.

35. Date, R. W.; Iglesias, E. F.; Rowe, K. E.; Elliott, J. M.; Bruce, D. W. Dalton Trans. 2003, 1914-1931. doi:10.1039/b212610a

36. Bruce, D. W.; Deschenaux, R.; Donnio, B.; Guillon, D. Organometallic Metallomesogens. In Comprehensive Organometallic Chemistry III; Crabtree, R. H.; Mingos, D. M. P., Eds.; Elsevier: Oxford, U.K., 2006; Vol. 12, Chapter 12.05, pp 195-294.

37. Porta, B.; Khamsi, J.; Noveron, J. C. Curr. Org. Chem. 2008, 12, 1298-1321. doi:10.2174/138527208785909565

38. Ghedini, M.; Aiello, I.; Crispini, A.; Golemme, A.; La Deda, M.; Pucci, D. Coord. Chem. Rev. 2006, 250, 1373-1390. doi:10.1016/j.ccr.2005.12.011

39. Rotkiewicz, K.; Grellmann, K. H.; Grabowski, Z. R. Chem. Phys. Lett. 1973, 19, 315-318. doi:10.1016/0009-2614(73)80367-7

40. Bhattacharyya, K.; Chowdhury, M. Chem. Rev. 1993, 93, 507-535. doi:10.1021/cr00017a022

41. Hicks, J.; Vandersall, M.; Babarogic, Z.; Eisenthal, K. B. Chem. Phys. Lett. 1985, 116, 18-24. doi:10.1016/0009-2614(85)80117-2

42. Golini, C. M.; Williams, B. W.; Foresman, J. B. J. Fluoresc. 1998, 8, 395-404. doi:10.1023/A:1020584801600

43. Dutta, A. K.; Kamada, K.; Ohta, K. J. Photochem. Photobiol., A: Chem. 1996, 93, 57-64. doi:10.1016/1010-6030(95)04140-0

44. Hazra, P.; Chakrabarty, D.; Chakraborty, A.; Sarkar, N. Chem. Phys. Lett. 2004, 388, 150-157. doi:10.1016/j.cplett.2004.02.078

45. Ghedini, M.; Pucci, D.; Crispini, A.; Bellusci, A.; La Deda, M.; Aiello, I.; Pugliese, T. Inorg. Chem. Commun. 2007, 10, 243-246. doi:10.1016/j.inoche.2006.10.032

46. Lo, K. K.-W.; Chung, C.-K.; Lee, T. K.-M.; Lui, L.-H.; Tsang, K. H.-K.; Zhu, N. Inorg. Chem. 2003, 42, 6886-6897. doi:10.1021/ic0346984

47. DePriest, J.; Zheng, G. Y.; Goswami, N.; Eichhorn, D. M.; Woods, C.; Rillema, D. P. Inorg. Chem. 2000, 39, 1955-1963. doi:10.1021/ic991306d

48. Simões, S.; Moreira, J. N.; Fonseca, C.; Düzgüneş, N.; Pedroso de Lima, M. C. Adv. Drug Delivery Rev. 2004, 56, 947-965. doi:10.1016/j.addr.2003.10.038

49. Pucci, D.; Bloise, R.; Bellusci, A.; Bernardini, S.; Ghedini, M.; Valentini, A.; Crispini, A. Mol. Cryst. Liq. Cryst. 2008, 481, 14-25. doi:10.1080/15421400701833981

50. Pucci, D.; Aiello, I.; Aprea, A.; Bellusci, A.; Crispini, A.; Ghedini, M. Chem. Commun. 2009, 1550-1552. doi:10.1039/b818603k

51. Newkome, G. R.; Patri, A. K.; Holder, E.; Schubert, U. S. Eur. J. Org. Chem. 2004, 235-254. doi:10.1002/ejoc.200300399

52. Pucci, D.; Barberio, G.; Crispini, A.; Francescangeli, O.; Ghedini, M.; La Deda, M. Eur. J. Inorg. Chem. 2003, 3649-3661. doi:10.1002/ejic.200300223
53. Evans, R. C.; Douglas, P.; Winscom, C. J. Coord. Chem. Rev. 2006, 250, 2093-2126. doi:10.1016/j.ccr.2006.02.007

54. Xu, X.; Liao, Y.; Yu, G.; You, H.; Di, C.; Su, Z.; Ma, D.; Wang, Q.; Li, S.; Wang, S.; Ye, J.; Liu, Y. Chem. Mater. 2007, 19, 1740-1748. doi:10.1021/cm062960b

55. Son, H.-J.; Han, W.-S.; Chun, J.-Y.; Kang, B.-K.; Kwon, S.-N.; Ko, J.; Han, S.-J.; Lee, C.; Kim, S. J.; Kang, S. O. Inorg. Chem. 2008, 47, 5666-5676. doi:10.1021/ic702491j

56. Barberio, G.; Bellusci, A.; Crispini, A.; Ghedini, M.; Golemme, A.; Prus, P.; Pucci, D. Eur. J. Inorg. Chem. 2005, 181-188. doi:10.1002/ejic.200400528

57. Binnemans, K. J. Mater. Chem. 2009, 19, 448-453. doi:10.1039/b811373d

58. Sapochak, L. S.; Burrows, P. E.; Garbuzov, V.; Ho, D. M.; Forrest, S. R.; Thompson, M. E. J. Phys. Chem. 1996, 100, 17766-17771. doi:10.1021/jp961770t

59. Tan, S.; Zhao, B.; Zou, Y.; Xiao, Z.; Wang, X.; Yu, G.; Liu, Y.; Zhu, D. J. Mater. Sci. 2004, 39, 1405-1406. doi:10.1023/B:JMSC.0000013904.51380.d7

60. Sapochak, L. S.; Padmaperuma, A.; Washton, N.; Endrino, F.; Schmett, G. T.; Marshall, J.; Fogarty, D.; Burrows, P. E.; Forrest, S. R. J. Am. Chem. Soc. 2001, 123, 6300-6307. doi:10.1021/ja010120m

61. Pucci, D.; Aiello, I.; Bellusci, A.; Crispini, A.; De Franco, I.; Ghedini, M.; La Deda, M. Chem. Commun. 2008, 2254-2256. doi:10.1039/b800030a

62. Crispini, A.; Aiello, I.; La Deda, M.; De Franco, I.; Amati, M.; Lelj, F.; Ghedini, M. Dalton Trans. 2006, 5124-5134. doi:10.1039/b606895b

\section{License and Terms}

This is an Open Access article under the terms of the Creative Commons Attribution License (http://creativecommons.org/licenses/by/2.0), which permits unrestricted use, distribution, and reproduction in any medium, provided the original work is properly cited.

The license is subject to the Beilstein Journal of Organic Chemistry terms and conditions:

(http://www.beilstein-journals.org/bjoc)

The definitive version of this article is the electronic one which can be found at: doi:10.3762/bjoc.5.54 